\title{
A CONSTRAINED SHAPE OPTIMIZATION PROBLEM IN ORLICZ-SOBOLEV SPACES
}

\author{
JOÃO VITOR DA SILVA, ARIEL M. SALORT, ANALÍA SILVA \\ $\&$ \\ JUAN F. SPEDALETTI
}

\begin{abstract}
In this manuscript we study the following optimization problem: given a bounded and regular domain $\Omega \subset \mathbb{R}^{N}$ we look for an optimal shape for the "W-vanishing window" on the boundary with prescribed measure over all admissible profiles in the framework of the Orlicz-Sobolev spaces associated to constant for the "Sobolev trace embedding". In this direction, we establish existence of minimizer profiles and optimal sets, as well as we obtain further properties for such extremals. Finally, we also place special emphasis on analyzing the corresponding optimization problem involving an " $\mathrm{A}-$ vanishing hole" (inside the domain) with volume constraint.
\end{abstract}

\section{INTRODUCTION}

1.1. A brief historic overview. Shape optimization problems constitute an important landmark concerning the modern development of the mathematical theory of optimization. Such issues are a longstanding subject of investigation, and currently they yet deserve increased attention by the academic community due to their intrinsic connection with several pivotal questions coming from pure and applied sciences. Some enlightening examples of such issues appear in eigenvalue problems with geometric constraints, optimization problems with constrained perimeter or volume, optimal design problems, problems in structural optimization, free boundary optimization problems, just to mention a few.

Heuristically, a shape optimization problem can be mathematically written as follows:

$$
\min \{\mathcal{J}(\mathcal{O}): \mathcal{O} \subset \Omega \text { with } \mathcal{O} \text { fulfilling a certain property } \mathbb{P}\},
$$

where $\Omega \subset \mathbb{R}^{N}$ is a bounded open set, $\mathcal{O}$ is an a priori unknown configuration (in general satisfying a specific property related to some constraint) and $\mathcal{J}$ is a "cost functional", which in several situations has an explicit integral representation, whose link with the competing configuration $\mathcal{O}$ arises via a solution of a PDE (cf. [7, 27] and 45] for nice surveys with a number of illustrative examples, we also recommend the reading of [3, [10, 11, [17, 20, 25], 21, 31, 35, 39] and [46] for other references with regard to free boundary and shape optimization problems).

2010 Mathematics Subject Classification. 35J60, 35J66, 35Q93, 46E30.

Key words and phrases. Nonlinear partial differential equations, Orlicz-Sobolev spaces, Shape optimization problems. 
In the scope of the modern Analysis and PDE's theory, the Sobolev Trace Embedding Theorem, namely

$$
W^{1, p}(\Omega) \hookrightarrow L^{q}(\partial \Omega)
$$

with the associated estimate for a constant $\mathcal{S}>0$ (Sobolev trace constant)

$$
\mathcal{S}\|u\|_{L^{q}(\partial \Omega)}^{p} \leq\|u\|_{W^{1, p}(\Omega)}^{p} \quad \text { (Sobolev trace inequality) }
$$

constitutes a fundamental tool in order to study certain issues in mathematics such as eigenvalue and Steklov type problems, functional type inequalities, existence and solvability of boundary-value problems among others.

Historically, optimization problems associated to the best constant for Sobolev trace embedding, namely

$$
\mathcal{S}_{p, q}=\inf \left\{\frac{\int_{\Omega}|\nabla u|^{p}+|u|^{p} d x}{\left(\int_{\partial \Omega}|u|^{q} d \mathcal{H}^{N-1}\right)^{\frac{p}{q}}}: u \in W^{1, p}(\Omega) \backslash W_{0}^{1, p}(\Omega)\right\},
$$

have received a warm attention by several authors in the last decades. The list of contributions is fairly diverse including aspects such as uniform bounds, symmetry properties, asymptotic behavior, local regularity of extremals and their free boundaries, approximations and many others (cf. [9, [12, [16, [18, [19, 21], 22, 23, 42 and 43 for more details). Particularly, we must highlight that in [12, [13], [18, 19] and 22] the authors studied for the $p$-Laplacian operator the problem of finding an optimal hole/window into the domain (resp. on the boundary) with prescribed measure associate to best constant for the Sobolev trace embedding. More precisely, they analyze the following quantity:

$$
\mathcal{S}(\Gamma)=\inf _{u \in \mathrm{X}_{\Gamma}} \frac{\int_{\Omega}|\nabla u|^{p}+|u|^{p} d x}{\left(\int_{\partial \Omega}|u|^{q} d \mathcal{H}^{N-1}\right)^{\frac{p}{q}}},
$$

where $1 \leq q<p_{\star}=\frac{p(N-1)}{N-p}$ (the critical exponent in the Sobolev trace embedding) and

$$
\mathrm{X}_{\Gamma}=\left\{u \in W^{1, p}(\Omega) \backslash W_{0}^{1, p}(\Omega) ; u=0 \mathcal{L}^{N} \text { - a.e. in } \Gamma\left(\text { resp. } \mathcal{H}^{N-1} \text { a.e. on } \partial \Gamma\right)\right\},
$$

where $\mathcal{L}^{N}$ (resp. $\mathcal{H}^{N-1}$ ) stands for the $N$-dimensional Lebesgue measure (resp. $(N-1)$-dimensional Hausdorff measure). Furthermore, another important issue in these works regards to the following shape optimization problem: for any fixed $0<\alpha<1$ the optimization problem

$$
\mathcal{S}(\alpha)=\inf \left\{\mathcal{S}(\Gamma): \Gamma \subset \Omega(\text { resp. } \Gamma \subset \partial \Omega) \text { s.t. } \frac{\mathcal{L}^{N}(\Gamma)}{\mathcal{L}^{N}(\Omega)}=\alpha\left(\text { resp. } \frac{\mathcal{H}^{N-1}(\Gamma)}{\mathcal{H}^{N-1}(\partial \Omega)}=\alpha\right)\right\}
$$

is achieved by a pair $\left(u_{0}, \Gamma_{0}\right)$ (an existence result). Moreover, under suitable regularity assumptions on the boundary, they obtain that $\Gamma_{0}=\{u=0\}$ (an explicit characterization result).

In the same way that in the classical Sobolev spaces, such trace embedding also plays a significant role in more general contexts governed by spaces with nonstandard growth, for which naturally we can quote the well-known Orlicz-Sobolev spaces (cf. [1, 8], 9] and [15] for such subjects). Such spaces extend the classical notion of Sobolev spaces to a context with non-power nonlinearities (cf. [5] and [40]), and currently such spaces are fully understood and studied in Analysis, PDE's, Free boundary problems, etc (cf. 22, 26, 30] and [41 for some surveys, and 24, [33], 35, 36] and [38 for some applications in PDE's theory). 
According to our knowledge, up to the date, there is no research concerning such optimization problems (S.E.C. ) in general sceneries with non-standard growth. For this very reason, such lack of investigations was one of our main starting points in considering shape optimization problems associate to the Sobolev trace embedding in the framework of Orlicz-Sobolev spaces.

1.2. Statement of main results. For the functional implementations in this article, we define the Orlicz-Sobolev embedding constant as follows:

(O.S.E.C.)

$$
S_{G, H}:=\inf _{u \in X} \frac{\int_{\Omega} G(|\nabla u|)+G(|u|) d x}{\int_{\partial \Omega} H(|u|) d \mathcal{H}^{N-1}},
$$

where $X=W^{1, G}(\Omega) \backslash W_{0}^{1, G}(\Omega)$ is an admissible functional class defined under Orlicz-Sobolev spaces, and $G$ and $H$ (throughout this manuscript) are suitable Young functions, both to be clarified a posteriori, see Section 2 for more details. It is worth highlighting that such a quantity O.S.E.C. is linked to some extent with the compact trace embedding

$$
W^{1, G}(\Omega) \hookrightarrow L^{H}(\partial \Omega)
$$

where $H$ and $G$ fulfill a certain compatibility condition, see Theorem 2.7 for details.

Different from the Rayleigh quotient in (S.E.C.), our definition employs an inhomogeneous quotient. This imposes an extra difficulty in our problem, which will be overcame by asking a "normalization" of boundary term (the associated modular), and then, we consider the class of admissible functions subject to such a constraint. Furthermore, we point out that different from its $p$-power counterpart (cf. [12, [19] and 22]), this version involves further extensions and difficulties that are treated and resolved throughout this manuscript.

In face of the previous statements, the first purpose of our manuscript consists in analyzing the shape optimization problem related to the "analogue" trace embedding constant associated to the Orlicz-Sobolev spaces. In this direction, we consider a regular and bounded domain $\Omega \subset \mathbb{R}^{N}$ and a subset of the boundary $\mathrm{W} \subset \partial \Omega$ (a "window") such that $\mathrm{W} \neq \partial \Omega$. Thus, we define the minimization problem

$$
S_{G, H}(\mathrm{~W}):=\inf \left\{\int_{\Omega} G(|\nabla u|)+G(|u|) d x: \int_{\partial \Omega} H(|u|) d \mathcal{H}^{N-1}=1\right\},
$$

where the infimum is taken over the set

$$
\mathrm{X}_{\mathrm{W}}:=\left\{u \in \mathrm{X}: u=0 \mathcal{H}^{N-1} \text { - a.e. on } \mathrm{W}\right\} .
$$

In our researches, the constant $S_{G, H}(\mathrm{~W})$ represents the counterpart for the first $H$-Steklov eigenvalue of the " $G$-Laplacian operator", which is defined as

$$
\Delta_{G} u:=\operatorname{div}\left(\frac{G^{\prime}(|\nabla u|)}{|\nabla u|} \cdot \nabla u\right) .
$$

Furthermore, notice that if $G(t)=H(t)=\frac{1}{p} t^{p}$ for $p>1$, then we fall into the well-known case of the Steklov eigenvalue for the $p$-Laplacian operator.

Next, let $0<\alpha<\mathcal{H}^{N-1}(\partial \Omega)$ be a fixed constant. Taking into account (Min), we define the following shape optimization problem:

$(\alpha-$ Window $) \quad \mathcal{S}_{G, H}(\alpha):=\inf \left\{S_{G, H}(W): W \subset \partial \Omega\right.$ and $\left.\mathcal{H}^{N-1}(W)=\alpha\right\}$. 
In this framework, a set $W \subset \partial \Omega$ in which the above infimum is achieved so-called optimal window for the constant $\mathcal{S}_{G, H}(\alpha)$.

Our first result provides the existence of minimizers and optimal shapes for our optimization problem, with a lower bound estimate for the null set of minimizers.

Theorem 1.1. Let $0<\alpha<\mathcal{H}^{N-1}(\partial \Omega)$ be a fixed quantity. Then

(1) There exists a function $u_{0} \in \mathrm{X}$ with $\mathcal{H}^{N-1}\left(\left\{u_{0}=0\right\}\right) \geq \alpha$ such that

$$
\mathcal{S}_{G, H}(\alpha)=\int_{\Omega} G\left(\left|\nabla u_{0}\right|\right)+G\left(\left|u_{0}\right|\right) d x .
$$

(2) There exists a set $\mathrm{W}_{0} \subset \partial \Omega$ such that

$$
\mathcal{H}^{N-1}\left(\mathrm{~W}_{0}\right)=\alpha \quad \text { and } \quad \mathcal{S}_{G, H}(\alpha)=S_{G, H}\left(\mathrm{~W}_{0}\right) .
$$

In contrast with previous result, now we establish that minimizers have an $\alpha$-sharp measure provided we assume enough regularity on the boundary.

Theorem 1.2. Suppose that the assumptions from Theorem 1.1 are in force. If $\partial \Omega$ is an enough regular set, let us say $C^{1, \beta}$, then $\mathcal{H}^{N-1}\left(\left\{u_{0}=0\right\}\right)=\alpha$.

It is worth to mention that any optimization pair $(u, \mathrm{~W})$ coming from Theorem 1.1 is linked thought the following singular/degenerate elliptic PDE:

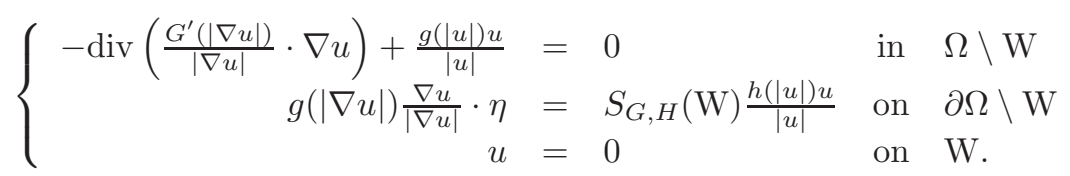

In comparison to previous results in the literature, namely [12, 21] and 22, our results include the case where the PDE/Neumann condition become "singular".

In final part of article, we put special attention to the shape optimization problem for finding an optimal interior hole $A \subseteq \Omega$ with prescribed volume associated to the "Orlicz-Sobolev embedding" constant, i.e.,

$$
\mathbb{S}_{G, H}(\mathrm{~A}):=\inf \left\{\int_{\Omega} G(|\nabla u|)+G(|u|) d x: \int_{\partial \Omega} H(|u|) d \mathcal{H}^{N-1}=1\right\},
$$

where the infimum is taken in the class

$$
\mathrm{X}_{\mathrm{A}}:=\left\{u \in W^{1, G}(\Omega) \backslash W_{0}^{1, G}(\Omega): u=0 \text { a.e. in } \mathrm{A}\right\} .
$$

In the same way we can consider an optimal design problem associated to the constant $\mathbb{S}_{G, H}(A)$, as follows: for $\alpha \in\left(0, \mathcal{L}^{N}(\Omega)\right)$ we define

$$
(\alpha \text {-Hole }) \quad \mathbb{S}(\alpha)=\inf \left\{\mathbb{S}_{G, H}(\mathrm{~A}): \mathrm{A} \subset \Omega \text { and } \mathcal{L}^{N}(\mathrm{~A})=\alpha\right\} .
$$

A set $A \subset \Omega$ in which the above infimium is achieved is called optimal interior hole.

The following result is the analogous one of Theorems 1.1 and 1.2 for the optimization of a hole into the domain instead on the boundary. The case of the $p$-Laplacian operator with Steklov boundary condition it was considered in [22].

Theorem 1.3. Given $0<\alpha<\mathcal{L}^{N}(\Omega)$. There exists a set $\mathrm{A}_{0} \subset \Omega$ such that $\mathcal{L}^{N}\left(\mathrm{~A}_{0}\right)=\alpha$ and $\mathbb{S}_{G, H}\left(\mathrm{~A}_{0}\right)=\mathbb{S}(\alpha)$. Moreover, every corresponding extremal $u_{0}$ to $\alpha$-Hole verifies that $\mathcal{L}^{N}\left(\left\{u_{0}=0\right\}\right)=\alpha$. 
In the next result we prove that there is no upper bound for $\mathbb{S}_{G, H}(\mathrm{~A})$, where A $\subset \Omega$ is an optimal interior hole.

Theorem 1.4. Let $0<\alpha<\mathcal{L}^{N}(\Omega)$ be a fixed quantity. Then, the following statement holds true:

$$
\sup \left\{\mathbb{S}_{G, H}(\mathrm{~A}): \mathrm{A} \subset \Omega \text { and } \mathcal{L}^{N}(\mathrm{~A})=\alpha\right\}=+\infty .
$$

Next, in order to give sense to "Orlicz-Sobolev trace constant" for functions vanishing in a negligible subset (zero $N$-dimentional Lebesgue measure) we will need to consider the space

$$
W_{\mathrm{A}}^{1, G}(\Omega)=\overline{C_{0}^{\infty}(\bar{\Omega} \backslash \mathrm{A})}
$$

where the closure is taken in $W^{1, G}$-norm, i.e., $W_{\mathrm{A}}^{1, G}(\Omega)$ are the functions that can be approximated by smooth functions that vanish in a neighborhood of A (compare with [26. Theorem 7.1.7]).

In this context the "Orlicz-Sobolev constant" is defined as

$$
\mathbb{S}_{\mathrm{A}}=\inf _{W_{A}^{1, G}(\Omega)}\left\{\int_{\Omega} G(|u|)+G(|\nabla u|) d x: \int_{\partial \Omega} H(|u|) d \mathcal{H}^{N-1}=1\right\} .
$$

At this point, it is important to question when $\mathbb{S}_{\mathrm{A}}$ recovers the usual "OrliczSobolev trace constant", i.e., when $\mathbb{S}_{\mathrm{A}}=\mathbb{S}_{\emptyset}$. A key ingredient for this result is the notion of $G$-capacitary sets (see section 6 for more details). We prove the following necessary and sufficient condition to this to hold.

Theorem 1.5. $\mathbb{S}_{\mathrm{A}}=\mathbb{S}_{\emptyset}$ if and only if $\mathrm{Cap}_{G}(\mathrm{~A})=0$.

Next, we address the continuity of $\mathbb{S}_{\mathrm{A}}$ with respect to $\mathrm{A}$ in the Hausdorff topology. Furthermore, we establish the continuity of corresponding extremals (in the $W^{1, G}$ norm) with respect to the Hausdorff topology of the sets A.

Theorem 1.6. Let $\mathrm{A}, \mathrm{A}_{\mathrm{k}}$ be closed sets such that

$$
\operatorname{dist}_{\mathcal{H}}\left(\mathrm{A}, \mathrm{A}_{\mathrm{k}}\right) \rightarrow 0 \quad \text { as } \quad k \rightarrow \infty .
$$

Then,

$$
\left|\mathbb{S}_{\mathrm{A}_{\mathrm{k}}}-\mathbb{S}_{\mathrm{A}}\right| \rightarrow 0 \quad \text { as } \quad k \rightarrow \infty .
$$

Moreover, if $u_{k}$ is an extremal for $\mathbb{S}_{\mathrm{A}_{\mathrm{k}}}$ normalized such that $\int_{\partial \Omega} H\left(\left|u_{k}\right|\right) d \mathcal{H}^{N-1}=1$, then up to a subsequence,

$$
u_{k} \rightarrow u \quad \text { strongly in } W_{A}^{1, G}(\Omega)
$$

and $u$ is an extremal for $\mathbb{S}_{\mathrm{A}}$.

In conclusion, a natural issue is what can be inferred about the extremals $u$ and "the optimal set" $\{u=0\} \subset \partial \Omega$ when the domain has certain symmetry. In our last result, we prove that (when $\Omega$ is a unity ball) there exists an extremal (resp. an optimal window) spherically symmetric.

Theorem 1.7. Let $\Omega=B_{1}$ and let $0<\alpha<\mathcal{H}^{N-1}(\partial \Omega)$ fixed. Then, there exists an optimal window which is a spherical cap. 
Organization of the paper. Our manuscript is organized as follows: in Section 2 we collect some preliminary results in the framework of Orlicz-Sobolev spaces. In Section 3 we present a number of properties for minimizers of our optimization problem. Section 4 is devoted to analyze our shape optimization problem and its features. In Sections 5 and 6 we establish existence and further results for extremals and optimal sets for the corresponding A-vanishing optimization problem. Finally, Section 7 is dedicated to prove a spherical symmetrization result.

\section{TECHNICAL TOOLS}

In this section we introduce some well-known definitions and auxiliary results.

2.1. Young functions. We consider the well-known set of Young functions. A function $G: \mathbb{R}^{+} \rightarrow \mathbb{R}$ in this class admits the following representation

$$
G(t)=\int_{0}^{t} g(s) d s, \quad t \geq 0,
$$

where $g:[0, \infty) \rightarrow[0, \infty)$ has the following properties:

(i) $g(0)=0$,

(ii) $g(s)>0$ for $s>0$,

(iii) $g$ is right continuous at any point $s \geq 0$,

(iv) $g$ is nondecreasing on $(0, \infty)$.

The following lemma provides several useful properties on Young functions.

Lemma 2.1. 26. Lemma 3.2.2]. A Young function $G$ is continuous, nonnegative, strictly increasing and convex on $[0, \infty)$. Moreover,

(i) $G(0)=0$ and $\lim _{t \rightarrow+\infty} G(t)=\infty$;

(ii) $\lim _{t \rightarrow 0^{+}} \frac{G(t)}{t}=0$ and $\lim _{t \rightarrow+\infty} \frac{G(t)}{t}=\infty$.

For our purposes we consider Young functions satisfying the so-called $\Delta_{2}-$ condition.

Definition 2.2. A Young function $G$ satisfies the $\Delta_{2}$ condition (or doubling condition) if

$$
G(2 t) \leq C G(t)
$$

for all $t \geq 0$ for a fixed positive constant $C$.

In particular, (cf. [26, Theorem 3.4.4]) a Young function $G$ satisfies the $\Delta_{2}$-condition if and only if

$$
\limsup _{t \rightarrow \infty} \frac{t G^{\prime}(t)}{G(t)}<\infty
$$

It is worth mentioning that such a kind of growing condition appears naturally when studying Orlicz-Sobolev spaces. Furthermore, we must compare such a condition with one considered in the Lieberman's pioneering work [33,

$$
g^{-}-1 \leq \frac{t g^{\prime}(t)}{g(t)} \leq g^{+}-1 \quad \forall t>0,
$$


for constants $0<g^{-} \leq g^{+}<\infty$, which establishes regularity estimates for weak solutions in Orlicz-Sobolev spaces. In fact, it is straightforward to see that such a condition implies that

$$
g^{-} \leq \frac{t G^{\prime}(t)}{G(t)} \leq g^{+} \quad \forall t>0 .
$$

Finally, the following version of the triangle inequality for Young functions holds.

Lemma 2.3 (24, Lemma 2.6]). Let $G$ be a Young function. Then for every $\eta>0$ there exists $C_{\eta}>0$ such that

$$
G(a+b) \leq C_{\eta} G(a)+(1+\eta)^{g^{+}} G(b) \quad a, b>0 .
$$

Example 2.4. Some well-known examples of Young functions include the following:

(1) $G(t)=t^{p}$ for $p>1$;

(2) $G(t)=t^{p}(\mathfrak{a}|\log t|+\mathfrak{b})$ for $p>1$ and $\mathfrak{a}, \mathfrak{b}>0$;

(3) $G(t)=\frac{t^{p}}{\mathfrak{a} \log (t+e)+\mathfrak{b}}$ for $p>1$ and $\mathfrak{a}, \mathfrak{b}>0$;

(4) If $G_{1}$ and $G_{2}$ are Young functions, then the composition $G(t)=\left(G_{1} \circ G_{2}\right)(t)$ is also a Young function;

(5) Finite linear combinations of Young functions with non-negative coefficients are also Young functions;

(6) The maximum among a finite family of Young functions is also a Young function.

2.2. Orlicz-Sobolev spaces and traces. Given a Young function $G$ and a bounded open set $\Omega$ we consider the spaces $L^{G}(\Omega)$ and $W^{1, G}(\Omega)$ defined as follows:

$$
\begin{aligned}
L^{G}(\Omega) & :=\left\{u: \mathbb{R} \rightarrow \mathbb{R} \text { measurable such that } \Phi_{G, \Omega}(u)<\infty\right\}, \\
W^{1, G}(\Omega) & :=\left\{u \in L^{G}(\Omega) \text { such that } \Phi_{G, \Omega}(|\nabla u|)<\infty\right\},
\end{aligned}
$$

where $\nabla u$ is considered in the distributional sense and the modular $\Phi_{G, \Omega}$ stands for

$$
\Phi_{G, \Omega}(u)=\int_{\Omega} G(|u|) d x .
$$

These spaces are endowed with the so-called Luxemburg norm defined as follows

$$
\|u\|_{L^{G}(\Omega)}=\inf \left\{\lambda>0: \Phi_{G, \Omega}\left(\frac{u}{\lambda}\right) \leq 1\right\}
$$

and

$$
\|u\|_{W^{1, G}(\Omega)}=\|u\|_{L^{G}(\Omega)}+\|\nabla u\|_{L^{G}(\Omega)} .
$$

It is worth highlighting that the spaces $L^{G}(\Omega)$ and $W^{1, G}(\Omega)$ are reflexive and separable Banach spaces if and only if $G$ and $\tilde{G}$ satisfy the $\Delta_{2}$-condition (cf. 2, Theorem 8.20], [26, Theorem 3.13.9] and [30, page 226], where

$$
\tilde{G}(t):=\sup _{s \geq 0}\{s t-G(s)\}
$$

denotes the complementary function (or Young conjugate) to $G$ (cf. 11, 8, 9, 15]). From now on, we will also assume that $\tilde{G}$ satisfies the $\Delta_{2}$-condition (cf. [26], Theorem 3.4.7] and [30, page 5]).

Now, we introduce the notion of weak solution. 
Definition 2.5. A function $u \in X_{W}$ is said to be a weak solution to

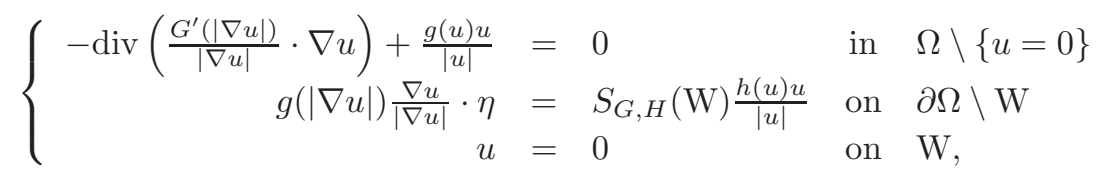

if

$$
\int_{\Omega} g(|\nabla u|) \frac{\nabla u}{|\nabla u|} \cdot \nabla \phi+\frac{g(u) u \phi}{|u|} d x=S_{G, H}(\mathrm{~W}) \int_{\partial \Omega} \frac{h(u) u \phi}{|u|} d \mathcal{H}^{N-1},
$$

for every test function $\phi \in C^{\infty}(\Omega)$. Here $\nabla u \cdot \eta$ is the outer unit normal derivative.

Remark 2.6. The following statements hold true:

(1) From the available regularity theory from [29] and [33, Ch.5], any minimizer $u_{0}$ of (Min) fulfills that $u_{0} \in C_{\text {loc }}^{1, \beta}(\Omega)$ for some $0<\beta<1$.

(2) From [32, if $\partial \Omega \backslash \overline{\mathrm{W}} \in C^{1, \gamma}$, then $u \in C^{1, \gamma}(\bar{\Omega} \backslash \mathrm{W})$ up to the boundary, for some $0<\gamma<1$.

(3) Notice that, if $u_{0}$ is minimizer to (Min), then $\left|u_{0}\right|$ as well. Hence, $v=\left|u_{0}\right|$ is a weak solution of

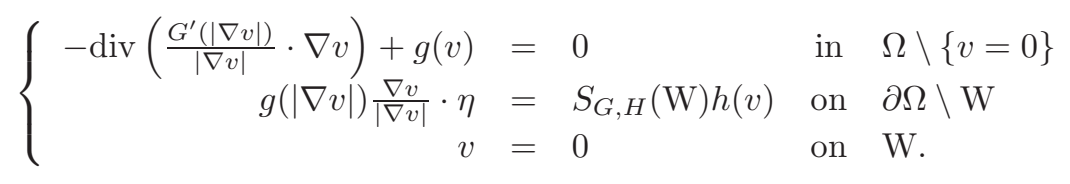

Thus, by using the maximum principle (cf. 38]), we obtain that $u_{0}$ does not change its sign. Consequently, we can always assume that

$$
u_{0}>0 \quad \text { in } \Omega \quad \text { and } \quad u_{0} \geq 0 \text { on } \partial \Omega .
$$

(4) From Hopf's Lemma (cf. [37, Theorem 1]) and boundary regularity results in 32 we obtain that any nonnegative solution $u_{0}$ to (2.2) satisfies

$$
u_{0}>0 \quad \text { in } \quad \bar{\Omega} \backslash \bar{W} .
$$

From now on, we will assume the following conditions on the Young function $G$ :

$$
\int_{0}^{1} \frac{G^{-1}(s)}{s^{1+\frac{1}{N}}} d s<\infty \text { and } \int_{1}^{\infty} \frac{G^{-1}(s)}{s^{1+\frac{1}{N}}} d s=\infty,
$$

which enable us to access to a compactness result regarding traces. For this purpose we recall that the Orlicz-Sobolev conjugate of $G$ (see [26, p. 352]) is defined as follows

$$
\left(G^{*}\right)^{-1}(t)=\int_{0}^{t} \frac{(G)^{-1}(s)}{s^{1+\frac{1}{N}}} d s .
$$

We say that $\mathfrak{A}$ increases more slowly than $\mathfrak{B}$ and denote $\mathfrak{A}<<\mathfrak{B}$ if and only if

$$
\lim _{t \rightarrow \infty} \frac{\mathfrak{A}(t)}{\mathfrak{B}(\lambda t)}=0
$$

for any $\lambda>0$.

Finally, the following result give us the compactness of the trace in the OrliczSobolev space $W^{1, G}(\Omega)$. 
Theorem 2.7 ([26, Theorem 7.4.6]). Let $\Omega \subset \mathbb{R}^{N}, N \geq 2$, be a $C^{0,1}$ domain. Let $G$ be a Young function satisfying the conditions (2.3), then the embedding

$$
W^{1, G}(\Omega) \hookrightarrow L^{H}(\partial \Omega)
$$

is compact for every Young function $H$ such that $H<<\Psi$, where

$$
\Psi(t)=\left(G^{*}(t)\right)^{\frac{N-1}{N}} .
$$

\section{Minimizers And optimal Shapes}

This section will be devoted to establish existence of extremals and optimal shapes for our optimization problem.

From now on, a set $\Omega \subset \mathbb{R}^{N}$ for $N \geq 2$, will denote a $C^{0,1}$ open bounded domain. The following result shows existence of minimizer for $S_{G, H}(\mathrm{~W})$.

Theorem 3.1 (Existence of minimizers). The constant $S_{G, H}(\mathrm{~W})$ in (Min is achieved for some function $u_{0} \in \mathrm{X}_{\mathrm{W}}$.

Proof. The proof follows as a consequence of the direct method in the calculus of variations. Indeed, take a minimizing sequence $\left\{u_{k}\right\}_{k \in \mathbb{N}}$ of $S_{G, H}(\mathrm{~W})$. That is

$$
S_{G, H}(\mathrm{~W})=\lim _{k \rightarrow \infty} \Phi_{G, \Omega}\left(\left|\nabla u_{k}\right|\right)+\Phi_{G, \Omega}\left(u_{k}\right),
$$

$\int_{\partial \Omega} H\left(\left|u_{k}\right|\right) d \mathcal{H}^{N-1}=1$, and $u_{k}=0 \mathcal{H}^{N-1}-$ a.e. on $\mathrm{W}$ for all $k \in \mathbb{N}$. The above limit tell us that there exists a positive constant $C$ such that

$$
\left\|u_{k}\right\|_{W^{1, G}(\Omega)} \leq C, \quad \forall k \in \mathbb{N} .
$$

Now, from the reflexivity of the space $W^{1, G}(\Omega)$, and by Theorem 2.7 there exists a function $u \in W^{1, G}(\Omega)$ such that, up to a subsequence,

$$
\begin{aligned}
& u_{k} \rightarrow u_{0} \text { weakly in } W^{1, G}(\Omega), \\
& u_{k} \rightarrow u_{0} \text { strongly in } L^{H}(\partial \Omega), \\
& u_{k} \rightarrow u_{0} \text { a.e. in } \partial \Omega \text { (see [26, p. 200] for details). }
\end{aligned}
$$

Note that (3.2) implies (see Remark (3.2))

$$
\int_{\partial \Omega} H\left(\left|u_{0}\right|\right) d \mathcal{H}^{N-1}=1
$$

Observe that the above implies that $u_{0}$ can not be zero over the complete boundary $\partial \Omega$, in this way $u_{0} \in W^{1, G}(\Omega) \backslash W_{0}^{1, G}(\Omega)$. Moreover, by (3.3) we get that $u_{0}=$ $0 \mathcal{H}$-a.e on W. Then,

$$
S_{G, H}(\mathrm{~W}) \leq \Phi_{G, \Omega}\left(\left|\nabla u_{0}\right|\right)+\Phi_{G, \Omega}\left(u_{0}\right) .
$$

Finally, from the convexity of $G$, the weakly lower semicontinuity of the application $v \mapsto \Phi_{G, \Omega}(|\nabla v|)+\Phi_{G, \Omega}(v)$ and the Fatou's lemma (cf. [14, Theorems 2.1.17, 2.2.8 and Lemma 2.3.16]) we get

$$
\begin{aligned}
\Phi_{G, \Omega}\left(\left|\nabla u_{0}\right|\right)+\Phi_{G, \Omega}\left(u_{0}\right) & \leq \liminf _{k \rightarrow \infty}\left[\Phi_{G, \Omega}\left(\left|\nabla u_{k}\right|\right)+\Phi_{G, \Omega}\left(u_{k}\right)\right] \\
& =S_{G, H}(\mathrm{~W}) .
\end{aligned}
$$

This proves the theorem. 
Remark 3.2. From [14, Lemma 2.1.14] we know that $\Phi_{H, \partial \Omega}\left(u_{k}\right)=1$ if and only if $\left\|u_{k}\right\|_{L^{H}(\partial \Omega)}=1$ for all $k$. Since $\lim _{k \rightarrow \infty}\left\|u_{k}\right\|_{L^{H}(\partial \Omega)}=\left\|u_{0}\right\|_{L^{H}(\partial \Omega)}$, we obtain that $\left\|u_{0}\right\|_{L^{H}(\partial \Omega)}=1$, and one more time using [14, Lemma 2.1.14] we conclude that $\Phi_{H, \partial \Omega}\left(u_{0}\right)=1$.

By following the approach from [22, Theorem 1.1], we will prove that $S_{G, H}(\mathrm{~W})$ is a lower semi-continuous map with respect to the window. As a result, we obtain existence of an optimal shape (window) for our optimization problem.

Theorem 3.3 (Existence of optimal shapes). Let $\left(\mathrm{W}_{\tau}\right)_{\tau>0} \subset \partial \Omega$ be a family of positive $\mathcal{H}^{N-1}$ - measurable subsets and $\mathrm{W}_{0} \subset \partial \Omega$ be a positive $\mathcal{H}^{N-1}$-measurable set, such that

$$
\chi_{W_{\tau}} \rightarrow \chi_{W_{0}} \quad * \text {-weakly in } L^{\infty}(\partial \Omega) .
$$

Then,

$$
S_{G, H}\left(\mathrm{~W}_{0}\right) \leq \liminf _{\tau \rightarrow 0+} S_{G, H}\left(\mathrm{~W}_{\tau}\right)
$$

Proof. Let $\left(\mathrm{W}_{\mathrm{k}}\right)_{k \in \mathbb{N}} \subset\left(\mathrm{W}_{\tau}\right)_{\tau>0}$ be a subsequence such that

$$
\mathcal{W}=\liminf _{\tau \rightarrow 0+} S_{G, H}\left(\mathrm{~W}_{\tau}\right)=\lim _{k \rightarrow+\infty} S_{G, H}\left(\mathrm{~W}_{k}\right) .
$$

Now, for each $k \in \mathbb{N}$, we consider $u_{k} \in \mathrm{X}_{\mathrm{W}_{k}}$ a non-negative minimizer of $S_{G, H}\left(\mathrm{~W}_{k}\right)$. Consequently, $\left(u_{k}\right)_{k \in \mathbb{N}}$ is bounded in $W^{1, G}(\Omega)$. Now, from reflexivity of the space $W^{1, G}(\Omega)$ and Theorem (2.7), there exists a function $u_{0} \in W^{1, G}(\Omega)$ such that, up to a subsequence,

$$
\begin{aligned}
& u_{k} \rightarrow u_{0} \text { weakly in } W^{1, G}(\Omega), \\
& u_{k} \rightarrow u_{0} \text { strongly in } L^{H}(\partial \Omega), \\
& u_{k} \rightarrow u_{0} \text { a.e. in } \partial \Omega \text { (See [26, Pag. 200] for details). }
\end{aligned}
$$

Particularly, $u_{0}$ is a non-negative profile with $\Phi_{H, \partial \Omega}\left(u_{0}\right)=1$ and using [14, Theorems 2.1.17, 2.2.8 and Lemma 2.3.16]

$$
\Phi_{G, \Omega}\left(\left|\nabla u_{0}\right|\right)+\Phi_{G, \Omega}\left(u_{0}\right) \leq \liminf _{k \rightarrow \infty}\left[\Phi_{G, \Omega}\left(\left|\nabla u_{k}\right|\right)+\Phi_{G, \Omega}\left(u_{k}\right)\right] .
$$

Furthermore, for each $k \in \mathbb{N}, u_{k}=0 \mathcal{H}^{N-1}$-a.e. on $\mathrm{W}_{k}$. From the convergence

$$
\chi_{W_{\tau}} \rightarrow \chi_{\mathrm{W}_{0}} * \text {-weakly in } L^{\infty}(\partial \Omega)
$$

and (3.6) we conclude that

$$
\int_{\mathrm{W}_{0}} u_{0}(x) d \mathcal{H}^{N-1}=\lim _{k \rightarrow+\infty} \int_{\mathrm{W}_{k}} u_{k}(x) d \mathcal{H}^{N-1}=0 .
$$

Since $u_{0}$ is a non-negative profile we conclude that $u_{0}=0 \quad \mathcal{H}^{N-1}$-a.e on $\mathrm{W}_{0}$. Therefore, $u_{0}$ is an admissible profile in the characterization of $S\left(\mathrm{~W}_{0}\right)$ and

$$
\begin{aligned}
S_{G, H}\left(\mathrm{~W}_{0}\right) & \leq \Phi_{G, \Omega}\left(\left|\nabla u_{0}\right|\right)+\Phi_{G, \Omega}\left(u_{0}\right) \\
& \leq \liminf _{k \rightarrow \infty}\left[\Phi_{G, \Omega}\left(\left|\nabla u_{k}\right|\right)+\Phi_{G, \Omega}\left(u_{k}\right)\right] \\
& =\mathcal{W},
\end{aligned}
$$

which finishes the proof. 
Remark 3.4. The pair $\left(u_{0}, S_{G, H}(\mathrm{~W})\right)$ from Theorem 3.1 is a solution, in the sense of Definition 2.5, of the following boundary value problem:

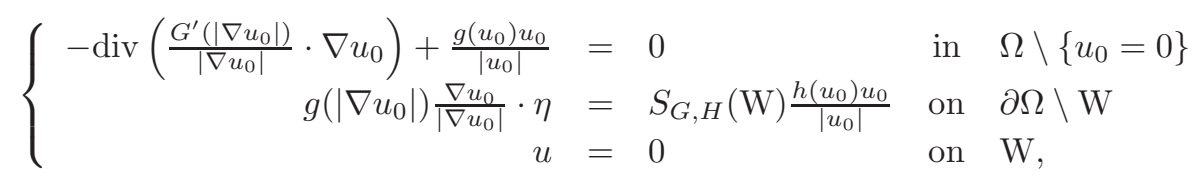

where $G^{\prime}(t)=g(t)$ and $H^{\prime}(t)=h(t)$.

Finally, observe that when $G(t)=H(t)=\frac{t^{p}}{p}$ with $p>1$, the last equation becomes the usual Steklov eigenvalue problem.

\section{The Shape OPtimization PROBLEM: OPTIMAL BOUNDARY WINDOW}

This section is devoted to provide the proofs of Theorem 1.1 and Theorem 1.2

Taking into account the shape optimization problem $\alpha$-Window the next result provides a characterization for the constant $\mathcal{S}_{G, H}(\alpha)$.

Lemma 4.1. The following characterization holds true

$$
\mathcal{S}_{G, H}(\alpha):=\inf \left\{\Phi_{G, \Omega}(|\nabla u|)+\Phi_{G, \Omega}(u): \Phi_{H, \partial \Omega}(u)=1, \mathcal{H}^{N-1}(\{u=0\}) \geq \alpha\right\},
$$

where the infimum is taken for functions in $W^{1, G}(\Omega) \backslash W_{0}^{1, G}(\Omega)$.

Proof. Let us define

$$
\widetilde{\mathcal{S}_{G, H}}(\alpha):=\inf \left\{\Phi_{G, \Omega}(|\nabla u|)+\Phi_{G, \Omega}(u): \Phi_{H, \partial \Omega}(u)=1, \mathcal{H}^{N-1}(\{u=0\}) \geq \alpha\right\} .
$$

Under such a definition, we will prove that $\widetilde{\mathcal{S}_{G, H}}(\alpha)$ is equivalent to (Min).

Firstly, we will prove that $\widetilde{\mathcal{S}_{G, H}}(\alpha) \leq \mathcal{S}_{G, H}(\alpha)$. Let $\mathrm{W} \subseteq \partial \Omega$ be such that $\mathcal{H}^{N-1}(\mathrm{~W}) \geq \alpha$ and let $u \in \mathrm{X}_{\mathrm{W}}$ be a nonnegative extremal of $S_{G, H}(\mathrm{~W})$. It is easy to verify that $u$ is an admissible function in $\widetilde{\mathcal{S}_{G, H}}(\alpha)$, so

$$
\widetilde{\mathcal{S}_{G, H}}(\alpha) \leq \Phi_{G, \Omega}(|\nabla u|)+\Phi_{G, \Omega}(u)=\mathcal{S}_{G, H}(\mathrm{~W}) .
$$

Hence, it follows that $\widetilde{\mathcal{S}_{G, H}}(\alpha) \leq \mathcal{S}_{G, H}(\alpha)$.

Next, we establish the opposite inequality, namely $\mathcal{S}_{G, H}(\alpha) \leq \widetilde{\mathcal{S}_{G, H}}(\alpha)$. For this purpose, we consider $\left\{v_{k}\right\}_{k \in \mathbb{N}}$ to be a minimizing sequence for $\widetilde{\mathcal{S}_{G, H}}(\alpha)$, i.e.,

$$
\widetilde{\mathcal{S}_{G, H}}(\alpha)=\lim _{k \rightarrow \infty} \Phi_{G, \Omega}\left(\left|\nabla v_{k}\right|\right)+\Phi_{G, \Omega}\left(v_{k}\right) \quad \text { and } \quad \mathcal{H}^{N-1}\left(\left\{v_{k}=0\right\}\right) \geq \alpha .
$$

Now, for any $k \in \mathbb{N}$ we choose $\mathrm{W}_{k} \subset\left\{v_{k}=0\right\}$ such that $\mathcal{H}^{N-1}\left(\mathrm{~W}_{k}\right)=\alpha$ (it is possible due to regularity of the Hausdorff measure, see [34, Ch. 1]). Hence, we get

$$
\mathcal{S}_{G, H}(\alpha) \leq S_{G, H}\left(\mathrm{~W}_{k}\right) \leq \Phi_{G, \Omega}\left(\left|\nabla v_{k}\right|\right)+\Phi_{G, \Omega}\left(v_{k}\right) .
$$

By taking limit when $k \rightarrow \infty$, we conclude that $\mathcal{S}_{G, H}(\alpha) \leq \widetilde{\mathcal{S}_{G, H}}(\alpha)$ as desired.

Finally, we are in position to supply for the proof of Theorem 1.1 
Proof of Theorem 1.1. Firstly, we will prove statement 1 Let $\left\{v_{k}\right\}_{k \in \mathbb{N}}$ be a nonnegative minimizing sequence of $\mathcal{S}_{G, H}(\alpha)$, i.e,

$$
v_{k} \geq 0, \Phi_{H, \partial \Omega}\left(v_{k}\right)=1, \mathcal{H}^{N-1}\left(\left\{v_{k}=0\right\}\right) \geq \alpha
$$

and

$$
\lim _{k \rightarrow \infty} \Phi_{G, \Omega}\left(\left|\nabla v_{k}\right|\right)+\Phi_{G, \Omega}\left(v_{k}\right)=\mathcal{S}_{G, H}(\alpha)
$$

So, $\left\{v_{k}\right\}_{k \in \mathbb{N}}$ is bounded in $W^{1, G}(\Omega)$ then by the reflexivity of the space $W^{1, G}(\Omega)$ there exists $u_{0} \in W^{1, G}(\Omega)$ such that

$$
v_{k} \rightarrow u_{0} \quad \text { weakly in } W^{1, G}(\Omega) .
$$

And from Theorem (2.7) we know that

$$
\begin{aligned}
& v_{k} \rightarrow u_{0} \quad \text { strongly in } L^{G}(\Omega), \\
& v_{k} \rightarrow u_{0} \quad \text { strongly in } L^{H}(\partial \Omega) \text {, } \\
& v_{k} \rightarrow u_{0} \quad \mathcal{H}^{N-1} \text { a.e. on } \partial \Omega \text {. }
\end{aligned}
$$

From these limits we obtain that $\Phi_{H, \partial \Omega}\left(u_{0}\right)=1$ (see, again, Remark (3.2)) and

$$
\mathcal{H}^{N-1}\left(\left\{u_{0}=0\right\}\right) \geq \limsup _{n \rightarrow \infty} \mathcal{H}^{N-1}\left(\left\{v_{k}=0\right\}\right) \geq \alpha .
$$

Then, $u_{0}$ is an admissible function for $\mathcal{S}_{G, H}(\alpha)$ (according to Lemma 4.1), so

$$
\mathcal{S}_{G, H}(\alpha) \leq \Phi_{G, \Omega}\left(\left|\nabla u_{0}\right|\right)+\Phi_{G, \Omega}\left(u_{0}\right) .
$$

The another inequality easily follows from the weak convergence in $W^{1, G}(\Omega)$ and the weakly lower semi-continuity of the functional $v \mapsto \Phi_{G, \Omega}(|\nabla v|)+\Phi_{G, \Omega}(v)$, i.e.,

$$
\Phi_{G, \Omega}\left(\left|\nabla u_{0}\right|\right)+\Phi_{G, \Omega}\left(u_{0}\right) \leq \lim _{k \rightarrow \infty} \Phi_{G, \Omega}\left(\left|\nabla v_{k}\right|\right)+\Phi_{G, \Omega}\left(v_{k}\right)=\mathcal{S}(\alpha) .
$$

Therefore, the function $u_{0}$ fulfills statement 1 .

Next, we will prove that statement (11) implies statement (2). Indeed, from statement (1) we know that there exists $u_{0} \in \mathrm{X}$ such that

$$
\mathcal{H}^{N-1}\left(\left\{u_{0}=0\right\}\right) \geq \alpha \quad \text { and } \quad \mathcal{S}(\alpha)=\Phi_{G, \Omega}\left(\left|\nabla u_{0}\right|\right)+\Phi_{G, \Omega}\left(u_{0}\right) .
$$

From this and the regularity of the corresponding Hausdorff measure (see [34, Ch. $1])$, there exists a closed set $\mathrm{W}_{0} \subseteq\left\{x \in \partial \Omega: u_{0}(x)=0\right\}$ such that

$$
\mathcal{H}^{N-1}\left(\mathrm{~W}_{0}\right)=\alpha \text {. }
$$

Consequently, by using (Min we obtain that

$$
S_{G, H}\left(\mathrm{~W}_{0}\right) \leq \Phi_{G, \Omega}\left(\left|\nabla u_{0}\right|\right)+\Phi_{G, \Omega}\left(u_{0}\right)=\mathcal{S}_{G, H}(\alpha) \leq S_{G, H}\left(\mathrm{~W}_{0}\right) .
$$

Therefore, $\mathcal{S}_{G, H}(\alpha)=S_{G, H}\left(\mathrm{~W}_{0}\right)$, thereby finishing the proof.

In the next result, namely Theorem 1.2, we find that the set of zeros of the minimizers coincide exactly with the optimal window.

Proof of Theorem 1.2, Let $u_{0} \in \mathrm{X}$ be an extremal for $\mathcal{S}_{G, H}(\alpha)$, then, from Lemma 4.1 it fulfills that

$$
\mathcal{H}^{N-1}\left(\left\{u_{0}=0\right\}\right) \geq \alpha \quad \text { and } \quad \mathcal{S}_{G, H}(\alpha)=\Phi_{G, \Omega}\left(\left|\nabla u_{0}\right|\right)+\Phi_{G, \Omega}\left(u_{0}\right) .
$$

Suppose for sake of contradiction that

$$
\mathcal{H}^{N-1}\left(\left\{u_{0}=0\right\}\right)>\alpha .
$$


Since the Hausdorff measure $\mathcal{H}^{s}$ is Borel regular for $(0 \leq s<\infty)$ (cf. 34, Ch. 1]) there exists a closed set $\mathrm{W}_{0} \subset\left\{x \in \partial \Omega: u_{0}(x)=0\right\}$ such that

$$
\mathcal{S}_{G, H}(\alpha) \leq S_{G, H}\left(\mathrm{~W}_{0}\right) .
$$

On the other hand, note that $u_{0}$ is an admissible function in the characterization of $S_{G, H}\left(\mathrm{~W}_{0}\right)$, from where

$$
S_{G, H}\left(\mathrm{~W}_{0}\right) \leq \Phi_{G, \Omega}\left(\left|\nabla u_{0}\right|\right)+\Phi_{G, \Omega}\left(u_{0}\right) .
$$

Consequently, $\mathcal{S}_{G, H}(\alpha)=S_{G, H}\left(\mathrm{~W}_{0}\right)$, and so $u_{0}$ is also a minimizer of $S_{G, H}\left(\mathrm{~W}_{0}\right)$. Hence, $u_{0}$ verifies in the weak sense

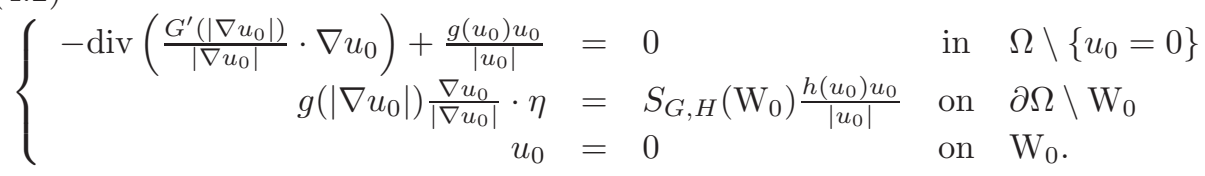

From the available regularity theory for $u_{0}$ (cf. 29] and [33, Ch. 5]) and Hopf type result (cf. 37, Theorem 1] for subsolutions) we conclude that

$$
\nabla u_{0} \cdot \eta>0 \text { on } \partial\left(\left\{x \in \partial \Omega: u_{0}(x)=0\right\} \backslash \mathrm{W}_{0}\right) \text { (point-wisely), }
$$

which contradicts the second equation in (4.2), thereby finishing the proof.

As a consequence from Theorem 1.2 we obtain the following monotonicity result:

Corollary 4.2. The set mapping $\alpha \mapsto \mathcal{S}_{G, H}(\alpha)$ is a strictly increasing function.

Proof. From definition, it holds that $\alpha \mapsto \mathcal{S}_{G, H}(\alpha)$ is a nondecreasing mapping. Next, suppose for sake of contradiction that there exists $0<\alpha<\sigma<\mathcal{H}^{N-1}(\partial \Omega)$ such that $\mathcal{S}_{G, H}(\alpha)=\mathcal{S}_{G, H}(\sigma)$. In particular, this would imply that minimizers for $\mathcal{S}_{G, H}(\sigma)$ are also minimizers for $\mathcal{S}_{G, H}(\alpha)$. However, if $u_{0}$ is a minimizer for $\mathcal{S}_{G, H}(\sigma)$, then from Theorem 1.2 we get

$$
\mathcal{H}^{N-1}\left(\left\{u_{0}=0\right\}\right)=\sigma>\alpha,
$$

which clearly contradicts Theorem 1.2 when applied for $\mathcal{S}_{G, H}(\alpha)$. Therefore, $\mathcal{S}_{G, H}$ is a strictly increasing map.

\section{Optimal interior holes: Proof of Theorems 1.3 and 1.4}

In this section we prove existence and some further properties on optimal holes with a prescribed volume.

The next proof runs similarly to [22, Theorem 1.2]. We will include the details for the reader convenience.

Proof of Theorem 1.3, Let $0<\alpha<\mathcal{L}^{N}(\Omega)$. It is easy to prove that $\mathbb{S}(\alpha)$ is obtained minimizing $\mathbb{S}_{G, H}(\mathrm{~A})$ over all the subsets $\mathrm{A} \subset \Omega$ such that $\mathcal{L}^{N}(\mathrm{~A}) \geq \alpha$. Moreover, it is clear that

$$
\inf \left\{\mathbb{S}_{G, H}(\mathrm{~A}): \mathrm{A} \subset \Omega, \mathcal{L}^{N}(\mathrm{~A})=\alpha\right\} \geq \inf \left\{\mathbb{S}_{G, H}(\mathrm{~A}): \mathrm{A} \subset \Omega, \mathcal{L}^{N}(\mathrm{~A}) \geq \alpha\right\} .
$$

Observe that test functions for a set of measure greater than or equal to $\alpha$ are also test functions for a set of measure $\alpha$, from where the two infimum above coincide. 
(5.1)

Now, let us prove that

$\mathbb{S}(\alpha)=\inf \left\{\Phi_{G, \Omega}(|\nabla u|)+\Phi_{G, \Omega}(u): u \in W^{1, G}(\Omega), \Phi_{H, \partial \Omega}(u)=1\right.$ and $\left.\mathcal{L}^{N}(\{u=0\}) \geq \alpha\right\}$.

Since the minimizer does not change sign, test functions can be considered to be nonnegative. As in the proof of Theorem 1.1 given a nonnegative minimizing sequence $\left\{v_{k}\right\}_{n \in \mathbb{N}}$ of $\mathbb{S}(\alpha)$ there exists $u_{0} \in W^{1, G}(\Omega)$ such that

$$
\begin{aligned}
& v_{k} \rightarrow u_{0} \text { weakly in } W^{1, G}(\Omega), \\
& v_{k} \rightarrow u_{0} \text { strongly in } L^{G}(\Omega), \\
& v_{k} \rightarrow u_{0} \text { strongly in } L^{H}(\partial \Omega), \\
& v_{k} \rightarrow u_{0} \text { a.e. in } \partial \Omega .
\end{aligned}
$$

Moreover, $\Phi_{H, \partial \Omega}\left(u_{0}\right)=1$ and $u_{0}$ can be assumed to be nonnegative.

Up to a subsequence, given the sets $\mathrm{A}_{k}=\left\{v_{k}=0\right\}$, there exists a function $0 \leq \phi \leq 1$ such that $\chi_{\mathrm{A}_{k}} \rightarrow \phi$ weakly in the dual space $L^{\tilde{G}}(\Omega)$. Particularly, for $\mathrm{A}=\{\varphi>0\}$ it holds that

$$
\mathcal{L}^{N}(\mathrm{~A}) \geq \int_{\Omega} \phi(x) d x=\lim _{k \rightarrow \infty} \int_{\Omega} \chi_{\mathrm{A}_{k}}(x) d x=\mathcal{L}^{N}\left(\mathrm{~A}_{k}\right) \geq \alpha .
$$

Since $u_{0}$ and $\phi$ are nonnegative, and

$$
\int_{\Omega} u_{0}(x) \phi(x) d x=\lim _{k \rightarrow \infty} \int_{\Omega} v_{k}(x) \chi_{\mathrm{A}_{k}}(x) d x=0,
$$

there holds that $u_{0}=0$ a.e. in $\mathrm{A}$. Then, $u_{0}$ is an admissible function for $\mathbb{S}(\alpha)$ and

$$
\mathbb{S}(\alpha) \leq \Phi_{G, \Omega}\left(\left|\nabla u_{0}\right|\right)+\Phi_{G, \Omega}\left(u_{0}\right) .
$$

The weak convergence in $W^{1, G}(\Omega)$ and the weakly lower semi-continuity of the functional $v \mapsto \Phi_{G, \Omega}(|\nabla v|)+\Phi_{G, \Omega}(v)$ gives that

$$
\Phi_{G, \Omega}\left(\left|\nabla u_{0}\right|\right)+\Phi_{G, \Omega}\left(u_{0}\right) \leq \lim _{k \rightarrow \infty} \Phi_{G, \Omega}\left(\left|\nabla v_{k}\right|\right)+\Phi_{G, \Omega}\left(v_{k}\right)=\mathbb{S}(\alpha)
$$

and therefore (5.1) holds.

Finally, it only remains to prove that $\mathcal{L}^{N}\left(\left\{u_{0}=0\right\}\right)=\alpha$. Suppose for the sake of contradiction that $u_{0} \equiv 0$ in a set $\mathrm{A}$ with $\mathcal{L}^{N}(\mathrm{~A})>\alpha$. By taking a subset we may assume that $\mathrm{A}$ is closed. Now, let $B$ be a small ball such that $\mathcal{L}^{N}(\mathrm{~A} \backslash B)>\alpha$ with $B$ centered in a point in $\partial \mathrm{A} \cap \partial \Omega_{1}$, where $\Omega_{1}$ is the connected component of $\Omega \backslash \mathrm{A}$ such that $\partial \Omega \subset \partial \Omega_{1}$. Notice that, we can pick the ball $B$ such that $\mathcal{L}^{N}(\mathrm{~A} \cap B)>0$. Particularly $\mathcal{L}^{N}\left(\left\{u_{0}=0\right\} \cap B\right)>0$.

Now, since $u_{0}$ is an extremal for $\mathbb{S}(\alpha)$ and $\mathcal{L}^{N}(\mathrm{~A} \backslash B)>\alpha$, it is also an extremal for $\mathbb{S}_{G, H}(A \backslash B)$. Thus,

$$
-\operatorname{div}\left(g\left(\left|\nabla u_{0}\right|\right) \frac{\nabla u_{0}}{\left|\nabla u_{0}\right|}\right)+\frac{g\left(u_{0}\right) u_{0}}{\left|u_{0}\right|}=0 \quad \text { in } \quad \Omega \backslash(\mathrm{A} \backslash B) .
$$

As $u_{0} \geq 0$, in view of Remark 2.6 (see item (3)), either $u \equiv 0$ or $u_{0}>0$ in each connected component of $\Omega \backslash(\mathrm{A} \backslash B)=(\Omega \backslash \mathrm{A}) \cup B$. Since $u_{0} \neq 0$ on $\partial \Omega$, in particular, $u_{0}>0$ in $B$, which clearly contradicts the choice of the ball $B$, and therefore $\mathcal{L}^{N}\left(\left\{u_{0}=0\right\}\right)=\alpha$. This concludes the proof. 
Now, we will establish that our optimization problem prevents the existence of maximal interior holes.

Proof of Theorem 1.4, For $0 \leq \varepsilon \ll 1$ fixed, let $\delta=\delta(\varepsilon)$ be such that

$$
\mathrm{A}_{\varepsilon, \delta}=\{x \in \Omega: \varepsilon \leq \operatorname{dist}(x, \partial \Omega) \leq \delta\}
$$

fulfills $\mathcal{L}^{N}\left(\mathrm{~A}_{\varepsilon, \delta}\right)=\alpha$. We affirm that

$$
\mathbb{S}_{G, H}\left(\mathrm{~A}_{\varepsilon, \delta}\right) \rightarrow+\infty \quad \text { as } \varepsilon \rightarrow 0^{+} .
$$

Indeed, let $v_{\varepsilon} \in W^{1, G}(\Omega) \backslash W_{0}^{1, G}(\Omega)$ be a minimizer for $\mathbb{S}_{G, H}\left(\mathrm{~A}_{\varepsilon, \delta}\right)$ according to definition (1.1) and remember that we assumed the normalization $\Phi_{H, \partial \Omega}\left(v_{\varepsilon}\right)=1$.

Now, for each $\sigma>0$ fixed, we consider

$$
\Omega_{\sigma}=\{x \in \Omega: \operatorname{dist}(x, \partial \Omega)>\sigma\} .
$$

Notice that $v_{\varepsilon}$ is a weak solution to

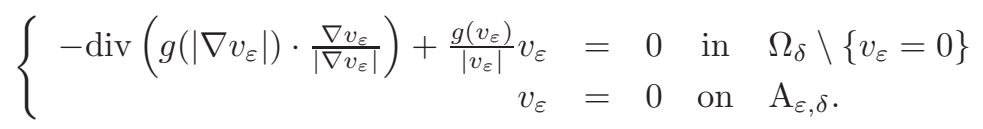

In particular, $v_{\varepsilon}=0$ on $\partial \Omega_{\delta}$. Hence, from Comparison Principle (cf. 38) $v_{\varepsilon}=0$ in $\Omega_{\delta}$. Furthermore, remember that by construction $v_{\varepsilon}=0$ in $\mathrm{A}_{\varepsilon, \delta}$, therefore

$$
v_{\varepsilon} \rightarrow 0 \text { a.e. } \Omega \text { as } \varepsilon \rightarrow 0 .
$$

Finally, supposing for the sake of contradiction that $\mathbb{S}_{G, H}\left(\mathrm{~A}_{\varepsilon, \delta}\right)$ is bounded. Then, up to a subsequence, there would exist a $u_{0} \in W^{1, G}(\Omega)$ such that

$$
\begin{array}{lrl}
v_{\varepsilon} \rightarrow u_{0} & \text { weakly in } & W^{1, G}(\Omega), \\
v_{\varepsilon} \rightarrow u_{0} & \text { strongly in } & L^{G}(\Omega), \\
v_{\varepsilon} \rightarrow u_{0} & \text { strongly in } & L^{H}(\partial \Omega), \\
v_{\varepsilon} \rightarrow u_{0} & \mathcal{H}^{N-1} \text { a.e. on } & \partial \Omega .
\end{array}
$$

Taking into account the sentences (5.2), (5.6), and the normalization condition $\Phi_{H, \partial \Omega}\left(v_{\varepsilon}\right)=1$ we obtain a contradiction.

\section{Proof of Theorems 1.5 and 1.6}

This section will deal with general shapes that may have zero Lebesgue measure. First, we analyze when the "Sobolev trace constant" perceives the set $\mathrm{A} \subset \mathbb{R}^{n}$ with zero Lebesgue measure. In this direction, we prove a continuity result of $\mathbb{S}_{\mathrm{A}}$ in relation to A in Hausdorff distance.

In order to study when $\mathbb{S}_{\mathrm{A}}$ recovers the usual Orlicz-Sobolev trace constant, i.e., when $\mathbb{S}_{\mathrm{A}}=\mathbb{S}_{\emptyset}$, we recall the notion of $G$-capacity for Young functions, which plays a fundamental role in Nonlinear Potential Theory.

Definition 6.1 (G-Capacity, 4, Definition 2.2]). Given a Young function $G$ satisfying the $\Delta_{2}$-condition, we define the $G$-capacity of $\mathrm{A} \subset \mathbb{R}^{N}$ by

$$
\operatorname{Cap}_{G}(\mathrm{~A})=\inf \left\{\int_{\mathbb{R}^{N}} G(\varphi)+G(|\nabla \varphi|) d x: \varphi \in W^{1, G}\left(\mathbb{R}^{N}\right) \cap C^{\infty}\left(\mathbb{R}^{N}\right) \text { and }\left.\varphi\right|_{\mathrm{A}} \geq 1\right\} .
$$

Moreover, if such a function does not exists, then $\operatorname{Cap}_{G}(\mathrm{~A})=\infty$. 
Next, we will deliver the proof of Theorem 1.5 .

Proof of Theorem 1.5. We prove both implications of the statement.

(1) Let us see that $\mathbb{S}_{\mathrm{A}}=\mathbb{S}_{\emptyset}$ implies that $\operatorname{Cap}_{G}(\mathrm{~A})=0$.

Given an extremal $u_{0}$ for $\mathbb{S}_{A}$, it is also an extremal for $\mathbb{S}_{\emptyset}$, and therefore it is a weak solution to

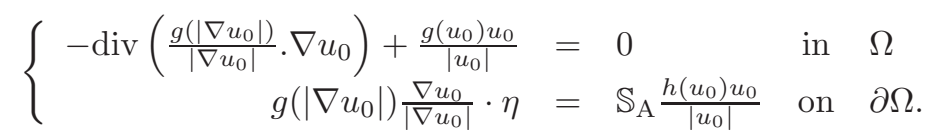

From Remark 2.6 items $(2,4)$, we have that $u_{0} \in C^{1, \gamma}(\bar{\Omega})$ and $u_{0}>0$ in $\bar{\Omega}$.

Since $u_{0} \in W_{\mathrm{A}}^{1, G}(\Omega)=\overline{C_{0}^{\infty}(\bar{\Omega} \backslash \mathrm{A})} \|^{\|\cdot\|_{W^{1, G}}}$, there exists a sequence $\left\{u_{k}\right\}_{k \in \mathbb{N}} \subset$ $C_{0}^{\infty}(\bar{\Omega} \backslash A)$ such that $u_{k} \rightarrow u_{0}$ in $W^{1, G}(\Omega)$.

Now, observe that the function $\varphi_{k}=1-\frac{u_{k}}{u_{0}}$ is identically equal to 1 in a neighborhood of A. Moreover, since $\beta=\inf \left\{u_{0}(x): x \in \bar{\Omega}\right\}>0$, we get

$$
\left\|\varphi_{k}\right\|_{L^{G}(\Omega)}=\left\|\frac{u_{0}-u_{k}}{u_{0}}\right\|_{L^{G}(\Omega)} \leq \frac{1}{\beta}\left\|u_{0}-u_{k}\right\|_{L^{G}(\Omega)}
$$

and

$$
\begin{aligned}
\left\|\nabla \varphi_{k}\right\|_{L^{G}(\Omega)} & =\left\|\frac{1}{u_{0}} \nabla u_{k}-\frac{u_{k}}{u_{0}^{2}} \nabla u_{0}\right\|_{L^{G}(\Omega)} \\
& =\left\|\frac{1}{u_{0}} \nabla\left(u_{k}-u_{0}\right)+\frac{1}{u_{0}^{2}} \nabla u_{0}\left(u_{0}-u_{k}\right)\right\|_{L^{G}(\Omega)} \\
& \leq \frac{1}{\beta}\left\|\nabla\left(u_{k}-u_{0}\right)\right\|_{L^{G}(\Omega)}+\frac{1}{\beta^{2}}\left\|\nabla u_{0}\right\|_{L^{\infty}(\Omega)}\left\|u_{0}-u_{k}\right\|_{L^{G}(\Omega)} .
\end{aligned}
$$

Therefore, for every $\varepsilon>0$ there exists $k_{0} \geq 1$ such that

$$
\left\|\varphi_{k}\right\|_{W^{1, G}(\Omega)}<\varepsilon
$$

if $k \geq k_{0}$. Finally, the proof finishes by using [26, Theorem 8.5.7] after extending $\varphi_{k}$ to $\mathbb{R}^{N}$ and regularizing.

(2) Let us see that $\operatorname{Cap}_{G}(\mathrm{~A})=0$ implies $\mathbb{S}_{\mathrm{A}}=\mathbb{S}_{\emptyset}$.

Let us prove that if $\operatorname{Cap}_{G}(\mathrm{~A})=0$ then

$$
W_{A}^{1, G}(\Omega)=W_{\emptyset}^{1, G}(\Omega)=W^{1, G}(\Omega),
$$

from where it will follows the lemma. Let $\mathrm{A} \subset \Omega$ be such that $\operatorname{Cap}_{G}(\mathrm{~A})=0$. Then, given $\varepsilon>0$ there exists $\varphi_{\varepsilon} \in W^{1, G}\left(\mathbb{R}^{N}\right) \cap C^{\infty}\left(\mathbb{R}^{N}\right)$ such that

$$
\int_{\mathbb{R}^{N}} G\left(\left|\varphi_{\varepsilon}\right|\right)+G\left(\left|\nabla \varphi_{\varepsilon}\right|\right) d x<\varepsilon
$$

and $\varphi_{\varepsilon} \equiv 1$ in a neighborhood of A.

Take $u \in C^{\infty}(\bar{\Omega})$ and define $u_{\varepsilon}=\left(1-\varphi_{\varepsilon}\right) u$. Observe that $u_{\varepsilon} \in W^{1, G}(\Omega)$ and $u_{\varepsilon}=0$ in A. Hence,

$$
\left\|u-u_{\varepsilon}\right\|_{W^{1, G}(\Omega)}=\left\|\varphi_{\varepsilon} u\right\|_{W^{1, G}(\Omega)} .
$$

Since $W_{\mathrm{A}}^{1, G}(\Omega)={\overline{C_{0}^{\infty}(\bar{\Omega} \backslash \mathrm{A})}}^{\|\cdot\|_{W^{1, G}}}$ the result follows if we show that $\left\|\varphi_{\varepsilon} u\right\|_{W^{1, G}(\Omega)}$ vanishes as $\varepsilon \rightarrow 0$. 
Now, since the $\Delta_{2}$ condition is in force, from [26, 3.10.4] the convergence in norm is equivalent to the convergence of modulars. Consequently, we have to show that

$$
\int_{\mathbb{R}^{N}} G\left(\left|\nabla\left(\varphi_{\varepsilon} u\right)\right|\right)+G\left(\left|\varphi_{\varepsilon} u\right|\right) d x \rightarrow 0 \quad \text { as } \varepsilon \rightarrow 0 .
$$

For that end, we have to bound each term of the expression

$$
\int_{\mathbb{R}^{N}} G\left(\left|\nabla\left(\varphi_{\varepsilon} u\right)\right|\right)+G\left(\left|\varphi_{\varepsilon} u\right|\right) d x \leq C \int_{\mathbb{R}^{N}} G\left(\left|u \nabla \varphi_{\varepsilon}\right|\right)+G\left(\left|\varphi_{\varepsilon} \nabla u\right|\right)+G\left(\left|\varphi_{\varepsilon} u\right|\right) d x .
$$

where we have used the $\Delta_{2}$ condition. First, since $G$ is increasing and the Sobolev extension theorem holds, using again the $\Delta_{2}$ condition,

$$
\int_{\mathbb{R}^{N}} G\left(\left|u \nabla \varphi_{\varepsilon}\right|\right) d x \leq \int_{\mathbb{R}^{N}} G\left(\left|\|u\|_{\infty} \nabla \varphi_{\varepsilon}\right|\right) d x \leq C_{\gamma}\|u\|_{\infty}^{\gamma} \int_{\mathbb{R}^{N}} G\left(\left|\nabla \varphi_{\varepsilon}\right|\right) d x \leq C_{\gamma}\|u\|_{\infty}^{\gamma} \varepsilon
$$

where $\gamma$ is a constant depending only on $g^{ \pm}$.

The second and third terms are bounded in the same way, and the proof concludes.

Before proving Theorem 1.6, let us recall the definition of Hausdorff distance.

Definition 6.2. Let $X, Y \subset \mathbb{R}^{N}$ be two non-empty subsets. The Hausdorff distance between $X$ and $Y$ is given by

$$
\operatorname{dist}_{\mathcal{H}}(X, Y):=\inf \left\{\varepsilon>0: X \subset Y_{\varepsilon} \text { and } Y \subset X_{\varepsilon}\right\},
$$

where $Z_{\varepsilon}=\bigcup_{z_{0} \in Z}\left\{z \in \mathbb{R}^{N} / \operatorname{dist}\left(z, z_{0}\right) \leq \varepsilon\right\}$ is the usual fattening of $Z$.

We are now in position to supply for the proof of Theorem 1.6

Proof of Theorem 1.6, Let $\mathrm{A}_{\varepsilon}=\bigcup_{x \in \mathrm{A}} B_{\varepsilon}(x)$. Assume that $\operatorname{dist}_{\mathcal{H}}\left(\mathrm{A}_{\mathrm{k}}, \mathrm{A}\right) \rightarrow 0$ as $k \rightarrow \infty$, then given $\varepsilon>0$ there exists $k_{0} \in \mathbb{N}$ such that $\mathrm{A}, \mathrm{A}_{\mathrm{k}} \subseteq A_{\varepsilon}$ if $k \geq k_{0}$ and it follows that

$$
W_{\mathrm{A}_{\varepsilon}}^{1, G}(\Omega) \subseteq W_{\mathrm{A}}^{1, G}(\Omega) \cap W_{\mathrm{A}_{\mathrm{k}}}^{1, G}(\Omega)
$$

First, note that

$$
\mathbb{S}_{\emptyset} \leq \mathbb{S}_{\mathrm{A}}, \mathbb{S}_{\mathrm{A}_{\mathrm{k}}} \leq \mathbb{S}_{\mathrm{A}_{\varepsilon}} \quad \text { if } k \geq k_{0} .
$$

Now, let $u \in W_{\mathrm{A}}^{1, G}(\Omega)$ be an extremal for $\mathbb{S}_{\mathrm{A}}$ normalized such that $\Phi_{H, \partial \Omega}(u)=1$. As $u \in W_{\mathrm{A}}^{1, G}(\Omega)=\overline{C_{0}^{\infty}(\bar{\Omega} \backslash \mathrm{A})} \|^{\|\cdot\|_{W^{1, G}}}$, given $\delta>0$ there exists $u_{\delta} \in C_{0}^{\infty}(\bar{\Omega} \backslash A)$ such that

$$
\left\|u-u_{\delta}\right\|_{W^{1, G}(\Omega)} \leq \delta .
$$

Moreover, we may suppose that

$$
\operatorname{supp}\left(u_{\delta}\right) \subseteq \bar{\Omega} \backslash \mathrm{A}_{\varepsilon}
$$


if $\varepsilon$ is small enough. Now $u_{\delta} \in W_{\mathrm{A}_{\mathrm{k}}}^{1, G}(\Omega)$ for $k \geq k_{0}$, so using Lemma 2.3

$$
\begin{aligned}
\mathbb{S}_{\mathrm{A}_{\mathrm{k}}} & \leq \Phi_{G, \Omega}\left(u_{\delta}\right)+\Phi_{G, \Omega}\left(\left|\nabla u_{\delta}\right|\right) \\
& \leq(1+\eta)^{g^{+}}\left[\Phi_{G, \Omega}(u)+\Phi_{G, \Omega}(|\nabla u|)\right]+C_{\eta}\left[\Phi_{G, \Omega}\left(u-u_{\delta}\right)+\Phi_{G, \Omega}\left(\left|\nabla\left(u-u_{\delta}\right)\right|\right)\right] \\
& \leq(1+\eta)^{g^{+}} \mathbb{S}_{\mathrm{A}}+C_{\eta}\left\|u-u_{\delta}\right\|_{W^{1, G}(\Omega)}^{g^{-}} \\
& \leq(1+\eta)^{g^{+}} \mathbb{S}_{\mathrm{A}}+C_{\eta} \delta^{g^{-}}
\end{aligned}
$$

where $\eta \geq 0$ is an arbitrary parameter and we have used that

$$
\Phi_{G, \Omega}\left(\frac{u-u_{\delta}}{\left\|u-u_{\delta}\right\|_{G}}\right) \leq 1 \Longrightarrow \Phi_{G, \Omega}\left(u-u_{\delta}\right) \leq\left\|u-u_{\delta}\right\|_{L^{G}(\Omega)}^{g^{-}} \leq\left\|u-u_{\delta}\right\|_{W^{1, G}(\Omega)}^{g^{-}} .
$$

Then, taking first limit as $\delta \rightarrow 0$, we obtain

$$
\mathbb{S}_{\mathrm{A}_{\mathrm{k}}} \leq(1+\eta)^{g^{+}} \mathbb{S}_{\mathrm{A}}
$$

and then, $\eta \rightarrow 0$, we get

$$
\mathbb{S}_{\mathrm{A}_{\mathrm{k}}} \leq \mathbb{S}_{\mathrm{A}}
$$

Analogously, it can be proved that the reverse inequality $\mathbb{S}_{\mathrm{A}} \leq \mathbb{S}_{\mathrm{A}_{\mathrm{k}}}$, and then

$$
\left|\mathbb{S}_{\mathrm{A}_{\mathrm{k}}}-\mathbb{S}_{\mathrm{A}}\right| \rightarrow 0 \text { as } k \rightarrow \infty .
$$

Let $u_{k}$ be an extremal for $\mathbb{S}_{\mathrm{A}_{\mathrm{k}}}$ normalized such that $\Phi_{G, H}\left(u_{k}\right)=1$. Then,

$$
\Phi_{G, \Omega}\left(u_{k}\right)+\Phi_{G, \Omega}\left(\left|\nabla u_{k}\right|\right)=\mathbb{S}_{\mathrm{A}_{\mathrm{k}}} \rightarrow \mathbb{S}_{\mathrm{A}} \quad \text { as } k \rightarrow \infty .
$$

It follows that $u_{k} \subseteq W^{1, G}(\Omega)$ is bounded. Hence, there exists a subsequence (still denoted by $u_{k}$ ) and a function $u \in W^{1, G}(\Omega)$ such that

$$
\begin{aligned}
& u_{k} \rightarrow u \text { weakly in } W^{1, G}(\Omega), \\
& u_{k} \rightarrow u \text { strongly in } L^{H}(\partial \Omega) .
\end{aligned}
$$

By definition of the spaces $W_{\mathrm{A}_{\mathrm{k}}}^{1, G}(\Omega)$ and $W_{\mathrm{A}}^{1, G}(\Omega)$ it is easy to see that $u \in W_{\mathrm{A}}^{1, G}(\Omega)$. Moreover, $\Phi_{G, H}(u)=1$ and also

$$
\begin{aligned}
\mathbb{S}_{\mathrm{A}} \leq \Phi_{G, \Omega}(u)+\Phi_{G, \Omega}(|\nabla u|) & \leq \lim _{k \rightarrow \infty} \Phi_{G, \Omega}\left(u_{k}\right)+\Phi_{G, \Omega}\left(\left|\nabla u_{k}\right|\right) \\
& =\lim _{k \rightarrow \infty} \Phi_{G, \Omega}\left(u_{k}\right)+\Phi_{G, \Omega}\left(\left|\nabla u_{k}\right|\right) \\
& \leq \lim _{k \rightarrow \infty} \mathbb{S}_{\mathrm{A}_{\mathrm{k}}}=\mathbb{S}_{\mathrm{A}} .
\end{aligned}
$$

The proof is concluded.

Example 6.3. Observe that when $H=G$ in our results, we can recover, to some extent the "Steklov eigenvalue problem". However, in contrast with the power case, for general Young functions it is not immediate that the hypothesis in Theorem 2.7 is fulfilled.

Let us see that, in fact, that such condition is satisfied, i.e, $G<<\left(G^{*}\right)^{\frac{N-1}{N}}$. Indeed, it is equivalent to $G^{\frac{N}{N-1}}<<G^{*}$. Moreover, since $G$ is increasing it also is equals to prove the following limit

$$
\lim _{t \rightarrow+\infty} \frac{\left(G^{*}\right)^{-1}(t)}{\left(G^{\frac{N}{N-1}}\right)^{-1}(t)}=0
$$

to hold (see [2, p.291]). 
First observe that integrating by part we obtain that

$$
\left(G^{*}\right)^{-1}(t)=\int_{0}^{t} \frac{G^{-1}(s)}{s^{1+\frac{1}{N}}} d s=N \int_{0}^{t} \frac{\left(G^{-1}(s)\right)^{\prime}}{s^{\frac{1}{N}}} d s-\frac{N}{t^{\frac{1}{N}}} G^{-1}(t) .
$$

We divide the previous expression by $\left(G^{\frac{N}{N-1}}\right)^{-1}(t)$, and analyze each one of the its two terms. Notice that $\left(G^{\frac{N}{N-1}}\right)^{-1}(t)=G^{-1}\left(t^{\frac{N-1}{N}}\right)$.

From the L'Hospital's rule, the first term behaves as follows

$$
\begin{aligned}
I_{1}:=\lim _{t \rightarrow \infty} \frac{N}{G^{-1}\left(t^{\frac{N-1}{N}}\right)} \int_{0}^{t} \frac{\left(G^{-1}(s)\right)^{\prime}}{s^{\frac{1}{N}}} d s & =\lim _{t \rightarrow \infty} \frac{\left(G^{-1}(t)\right)^{\prime}}{t^{\frac{1}{N}}} \frac{N}{\left(G^{-1}\left(t^{\frac{N-1}{N}}\right)\right)^{\prime}} \\
& =\lim _{t \rightarrow \infty} \frac{N}{t^{\frac{1}{N}}} \frac{G^{\prime}\left(G^{-1}\left(t^{\frac{N-1}{N}}\right)\right)}{G^{\prime}\left(G^{-1}(t)\right)} .
\end{aligned}
$$

It is not hard to prove that $G^{-1}$ satisfies a condition analogous to (2.1) with constants $\left(g^{+}\right)^{-1}$ and $\left(g^{-}\right)^{-1}$, see [36, Lemma 2.2]. Then

$$
\frac{N}{t^{\frac{1}{N}}} \frac{G^{\prime}\left(G^{-1}\left(t^{\frac{N-1}{N}}\right)\right)}{G^{\prime}\left(G^{-1}(t)\right)} \leq \frac{g^{+}}{g^{-}} \frac{N}{t^{\frac{1}{N}}} \frac{G\left(G^{-1}\left(t^{\frac{N-1}{N}}\right)\right)}{G\left(G^{-1}(t)\right)}=\frac{g^{+}}{g^{-}} \frac{N}{t^{\frac{2}{N}}} \frac{G^{-1}(t)}{G^{-1}\left(t^{1-\frac{1}{N}}\right)}
$$

but again, using the $\Delta_{2}$ condition for $G^{-1}$, see [36. Lemma 2.2], the above inequality can be bounded as follows

$$
\frac{g^{+}}{g^{-}} \frac{N}{t^{\frac{2}{N}}} \frac{G^{-1}(t)}{G^{-1}\left(t^{1-\frac{1}{N}}\right)}=\frac{g^{+}}{g^{-}} \frac{N}{t^{\frac{2}{N}}} \frac{G^{-1}\left(t^{1-\frac{1}{N}} t^{\frac{1}{N}}\right)}{G^{-1}\left(t^{1-\frac{1}{N}}\right)} \leq \frac{g^{+}}{g^{-}} \frac{N t^{\frac{1}{N g^{-}}}}{t^{\frac{2}{N}}}=\frac{g^{+}}{g^{-}} N t^{\frac{1}{N}\left(\frac{1}{g^{-}}-1\right)}
$$

for $t>1$. Consequently, since $g^{-}>1$, the last three expressions lead to $I_{1}=0$.

Let us deal with the second term. Using again the $\Delta_{2}$ condition for $G^{-1}$ we get

$$
I_{2}:=\lim _{t \rightarrow \infty} \frac{N}{t^{\frac{1}{N}}} \frac{G^{-1}(t)}{G^{-1}\left(t^{1-\frac{1}{N}}\right)}=\lim _{t \rightarrow \infty} \frac{N}{t^{\frac{1}{N}}} \frac{G^{-1}\left(t^{1-\frac{1}{N}} t^{\frac{1}{N}}\right)}{G^{-1}\left(t^{1-\frac{1}{N}}\right)} \leq \lim _{t \rightarrow \infty} N t^{\frac{1}{N}\left(\frac{1}{g^{-}}-1\right)}
$$

and it vanishes since $g^{-}>1$. Since $I_{1}+I_{2}=0$, 6.1) holds as required.

\section{SPHERICAL SYMMETRIZATION}

In this short section we will characterize the optimal window $\mathrm{W}_{0} \subset \partial \Omega$ in our optimization problem as a spherical cap provided that $\Omega=B_{1}$. For that purpose, an essential tool is played by the spherical symmetrization.

Given a mensurable set $\mathcal{E} \subset \mathbb{R}^{N}$, the spherical symmetrization $\mathcal{E}^{\sharp}$ of $\mathcal{E}$ with respect to an axis given by a unit vector $e_{k}$ reads as follows: for each positive number $r$, take the intersection $\mathcal{E} \cap \partial B(0, r)$ and replace it by the spherical cap of the same $\mathcal{H}^{N-1}$-measure and center $r e_{k}$. Hence, $\mathcal{E}^{\sharp}$ is the union of these caps.

Now, the spherical symmetrization $u^{\sharp}$ of a measurable function $u: \Omega \rightarrow \mathbb{R}_{+}$is constructed by symmetrizing the super-level sets so that, for all $t$

$$
\left\{u^{\sharp} \geq t\right\}=\{u \geq t\}^{\sharp} \text {. }
$$

We recommend to the reader references [28] and 44] for more details.

In the following, we recall some useful tools from Measure Theory. 
Definition 7.1. Let $(\mathbb{X}, \mathfrak{M}, \mu)$ be a measure space. Given a measurable function $f: \mathbb{X} \rightarrow \mathbb{R}$, the distribution function of $f$ is the function $\varrho_{f}:[0,+\infty) \rightarrow[0, \mu(\mathbb{X})]$ defined as follows:

$$
\varrho_{f}(t):=\mu(\{x \in \mathbb{X}:|f(x)|>t\}) .
$$

The next result holds as consequence from Fubini-Tonelli's theorem.

Lemma 7.2. Let $(\mathbb{X}, \mathfrak{M}, \mu)$ be a finite measure space, let $G \in C^{1}(\mathbb{R})$ a convex function and let $f: \mathbb{X} \rightarrow \mathbb{R}$ be a measurable function. Then,

$$
\int_{\mathbb{X}} G(|f(x)|) d \mu(x)=\int_{0}^{+\infty} G^{\prime}(t) \varrho_{f}(t) d t .
$$

Remark 7.3. Another important piece of information for our approach is the following result from [44, Proposition 5, item b]: if $u \in L^{1}(\mathbb{X})$ then on the Borel sets

$$
\varrho_{u}(t)=\varrho_{u^{\sharp}}(t) \quad \forall t \geq 0 .
$$

Next, for the characterization of the optimal window we need the following result.

Proposition 7.4. Let $u \in W^{1, G}\left(B_{1}\right) \cap W^{1, H}\left(\partial B_{1}\right)$ and $u^{\sharp}$ be its spherical symmetrization. Assume that

$$
P_{B_{1}}\left(\left\{x \in B_{1}: u(x)>t\right\}\right) \sqrt{1} \geq \gamma \varrho u^{\frac{N-1}{N}}(t),
$$

for some positive constant $\gamma \geq N \sqrt[N]{\omega_{N}}$ and any $t \geq 0$. Then, $u^{\sharp} \in W^{1, G}\left(B_{1}\right) \cap$ $W^{1, H}\left(\partial B_{1}\right)$. Moreover,

(1) $\int_{B_{1}} G\left(\left|u^{\sharp}\right|\right) d x=\int_{B_{1}} G(|u|) d x$,

(2) $\int_{\partial B_{1}} H\left(\left|u^{\sharp}\right|\right) d \mathcal{H}^{N-1}=\int_{\partial B_{1}} H(|u|) d \mathcal{H}^{N-1}$,

(3) $\int_{B_{1}} G\left(\left|\nabla u^{\sharp}\right|\right) d x \leq \int_{B_{1}} G(|\nabla u|) d x$.

Proof. The statements (10) and (2) hold by combining Lemma 7.2 and Remark 7.3

For the last statement, from [6, Section 3] we know that

$$
\int_{B_{1}} G\left(\frac{\left|\nabla u^{\sharp}\right|}{\lambda_{0}}\right) d x \leq \int_{B_{1}} G(|\nabla u|) d x
$$

for a constant $\lambda_{0}=\frac{N \sqrt[N]{\omega_{N}}}{\gamma}$. From assumption on $\gamma$ we obtain that $0<\lambda_{0} \leq 1$. In this case, from the convexity of $G$ and (7.1) we obtain that

$$
\int_{B_{1}} G\left(\left|\nabla u^{\sharp}\right|\right) d x \leq \lambda_{0} \int_{B_{1}} G\left(\frac{\left|\nabla u^{\sharp}\right|}{\lambda_{0}}\right) d x \leq \int_{B_{1}} G(|\nabla u|) d x,
$$

which concludes the proof.

Finally, we will present the proof of our symmetrization result.

\footnotetext{
${ }^{1}$ Here $P_{\Omega}$ means the perimeter, in the sense of De Giorgi, relative to $\Omega$. For such a concept in the general case, see 34 .
} 
Proof of Theorem 1.7. Firstly, for a fixed $\alpha \in\left(0, \mathcal{H}^{N-1}\left(\partial B_{1}\right)\right)$ Theorem (1.2) assures that there exists a profile $u_{0} \in \mathrm{X}_{\mathrm{W}}$ such that

$$
\mathcal{H}^{N-1}\left(\left\{u_{0}=0\right\}\right)=\alpha \quad \text { and } \quad \mathcal{S}_{G, H}(\alpha)=\Phi_{G, B_{1}}\left(\left|\nabla u_{0}\right|\right)+\Phi_{G, B_{1}}\left(u_{0}\right) .
$$

Now, let $u_{0}^{\sharp}$ be the spherical symmetrization of $u_{0}$. Notice that $u_{0}^{\sharp}$ is an admissible profile in the optimization process of $\mathcal{S}_{G, H}(\alpha)$, and by the Proposition (7.4)

$$
\mathcal{S}_{G, H}(\alpha) \leq \Phi_{G, B_{1}}\left(\left|\nabla u_{0}^{\sharp}\right|\right)+\Phi_{G, B_{1}}\left(u_{0}^{\sharp}\right) \leq \Phi_{G, B_{1}}\left(\left|\nabla u_{0}\right|\right)+\Phi_{G, B_{1}}\left(u_{0}\right)=\mathcal{S}_{G, H}(\alpha) .
$$

Therefore,

$$
\mathcal{S}_{G, H}(\alpha)=\Phi_{G, B_{1}}\left(\left|\nabla u_{0}^{\sharp}\right|\right)+\Phi_{G, B_{1}}\left(u_{0}^{\sharp}\right) .
$$

Finally, since

$$
\mathcal{H}^{N-1}\left(\mathrm{~W}_{0}\right)=\mathcal{H}^{N-1}\left(\left\{u_{0}=0\right\}\right)=\alpha,
$$

where $\mathrm{W}_{0}=\left\{x \in \partial B_{1}: u_{0}^{\sharp}(x)=0\right\}$, we conclude by using (7.2) that

$$
\mathcal{S}_{G, H}(\alpha)=S_{G, H}\left(\mathrm{~W}_{0}\right),
$$

which assures that $\mathrm{W}_{0}$ is an optimal window. As a direct consequence we obtain the desired symmetry result, because the optimal window $\mathrm{W}_{0}$ is a spherical cap.

Remark 7.5. At this point, the following question arises: if $\Omega$ is symmetric, does $A_{0}$ (in the case of optimal interior hole) inherit the symmetry of the domain? The answer is positive in some scenarios, for instance, as proved previously, if $\Omega=B_{1}$, then $\mathrm{A}_{0}$ is spherically symmetric (cf. 22], 10, 12] and [13] for similar results). However, for general configurations of the domain, $A_{0}$ is not necessarily radially symmetric (cf. [16]).

Acknowledgements. This paper was supported by grants PROICO 031906, UNSL, CONICET PIP 11220150100032CO and UBACyT 20020130100283BA. The authors would like to thank Prof. Julián Fernández Bonder for reading the draft this manuscript and providing insightful comments. J.V. da Silva would like to thank the Department of Mathematics and FCNyE from Universidad de Buenos Aires for providing an excellent working environment and scientific atmosphere during his Postdoctoral program. J.V. da Silva thanks also to IMASL (CONICET) and Department of Mathematics from Universidad Nacional de San Luis for their warm hospitality and for fostering a pleasant scientific atmosphere during his visit where part of this manuscript was written. J.V. da Silva, A.M. Salort and A. Silva are members of CONICET.

\section{REFERENCES}

[1] Adams, R. On the Orlicz-Sobolev lmbedding Theorem. J. Funct. Anal., 24 (1977), 241-257.

[2] Adams, R. and Fournier, J. Sobolev Spaces, Volume 140, 2nd Edition, Academic Press (2003).

[3] Aguilera, N., Alt, H. and Caffarelli, L. An optimization problem with volume constraint. SIAM J. Control Optim. 24, (1986), 191-198.

[4] Biegert. On a capacity for modular spaces. Journal of Mathematical Analysis and Applications. V. 358, 294-306.

[5] Birnbaum, Z. W. and Orlicz, W. Über die Verallgemeinerung des Begriffes der zueinander Konjugierten Potenzen, Studia Mathematica, 3, 1-67, (1931).

[6] Bramanti, M. On the gradient of Schwarz symmetrization of functions in Sobolev spaces. Boll. Un. Mat. Ital. B (7) 7 (1993), no. 2, 413-430.

[7] Bucur, D. and Buttazzo, G. Variational Methods in Shape Optimization Problems. Progress in Nonlinear Diff. Eq. and their Applications, 65. Birkhäuser Boston, Inc., 2005. viii+216 pp. 
[8] Cianchi, A. Continuity properties of functions from Orlicz-Sobolev spaces and embedding theorems. Ann. Sc. Norm. Super. Pisa Cl. Sci., tome 23, n. 3 (1996), p. 575-608.

[9] Cianchi, A. Orlicz-Sobolev boundary trace embeddings. Math. Z. (2010) 266: 431-449.

[10] da Silva, J.V., Del Pezzo, L. and Rossi, J. A limiting optimization problem with volume constraint and Neumann boundary conditions. arXiv preprint arXiv:1805.02633

[11] da Silva, J.V. and Rossi, J. The limit as $p \rightarrow \infty$ in free boundary problems with fractional $p$ Laplacians. To appear in Trans. Amer. Math. Soc. DOI: https://doi.org/10.1090/tran/7559.

[12] Del Pezzo, L., Fernández Bonder, J. and Neves, W. Optimal boundary holes for the Sobolev trace constant. J. Differential Equations, 251 (2011), no. 8, 2327-2351.

[13] Denzler, J. Windows of given area with minimal heat diffusion, Trans. Amer. Math. Soc. 351 (1999) 569-580.

[14] Diening, L., Harjulehto, P., Hästö, P. and M. Ružička. Lebesgue and Sobolev spaces with variable exponents. Lecture Notes in Mathematics, 2017. Springer, Heidelberg, 2011.

[15] Donaldson, T. K. and Trudinger, N. Orlics-Sobolev spaces and imbedding theorems. J. Funct. Anal. 8, 52-75, (1971).

[16] Fernández Bonder, J., Lami Dozo, E. and Rossi, J. Symmetry properties for the extremals of the Sobolev trace embedding. Ann. Inst. H. Poincaré Anal. Non Linéaire. 21 (2004), no. 6, 795-805.

[17] Fernández Bonder, J., Martínez, S. and Wolanski, N. An optimization problem with volume constraint for a degenerate quasilinear operator, J. Differential Equations 227 (2006) 80-101.

[18] Fernández Bonder, J., Orive, R. and and Rossi, J. The best Sobolev trace constant in domains with holes for critical or subcritical exponents. ANZIAM J., 49 (2007), 213-230.

[19] Fernández Bonder, J., Rossi, J. and Shönlieb. The best constant and extremals of the Sobolev embeddings in domains with holes: the $L^{\infty}$ case. Illinois J. Math., 52 (2008), no. 4, 1111-1121.

[20] Fernández Bonder, J., Rossi, J. and Spedaletti, J. Optimal design problems for the first p-fractional eigenvalue with mixed boundary conditions. Advanced Nonlinear Studies, 18(2), 323-335.

[21] Fernández Bonder, J., Rossi, J. and and Wolanski, N. Regularity of the free boundary in an optimization problem related to the best Sobolev trace constant. SIAM J. Control Optim., 44 (2005), no. 5, 1614-1635.

[22] Fernández Bonder, J., Rossi, J. and and Wolanski, N. On the best Sobolev trace constant and extremals in domains with holes. Bull. Sci. Math. 130 (2006), 565-579.

[23] Fernández Bonder, J. and Saintier, N. Estimates for the Sobolev trace constant with critical exponent and applications. Ann. Mat. Pura Appl. (4), 187 (2008), no. 4, 683-704.

[24] Fernández Bonder, J. and Salort, A. Fractional order Orlicz-Sobolev spaces. arXiv preprint arXiv: 1707.03267

[25] Fernández Bonder, J. and Spedaletti, J.F. Some nonlocal optimal design problems. J. Math. Anal. Appl., 459 (2018), no. 2, 906-931.

[26] Fucik, S., John, O. and Kufner, A. Function Spaces. (Mechanics: Analysis) Noordhoff International Publishing, Leyden and Academia, Praha, 1977.

[27] Henrot, A. and Pierre, M. Variation et optimization de forme: un analyse géométric. Mathematics and Applications 48, Springer, 2005.

[28] Kawohl, B. Rearrangements and Convexity of Level Sets in PDE. Lect. Notes in Math., vol. 1150, Springer-Verlag, Berlin, 1985.

[29] Korolev, A. G. On boundedness of generalized solutions of elliptic differential equations with nonpower nonlinearities. Mat. Sb. 180 (1989), 78-100 (in Russian).

[30] Krasnosel'skii, M. A. and Rutickii, Ya. B. (1961) Convex Functions and Odicz Spaces. (Noordhoff, Groningen, The Netherlands).

[31] Lederman, C. A free boundary problem with a volume penalization. Ann. Scuola Norm. Sup. Pisa Cl. Sci. (4) 23 (1996), 249-300.

[32] Lieberman, Gary M. Boundary regularity for solutions of degenerate elliptic equations. Nonlinear Anal. 12 (11) (1988) 1203-1219.

[33] Lieberman, Gary M. The natural generalization of the natural conditions of Ladyzhenskaya and Uralitseva for elliptic equations. Comm. Partial Diff. Eq., v. 16, 1991, n. 2-3, pp. 311-361.

[34] Lin, F-H. and Yang, X. Geometric measure theory: an introduction. (Advanced mathematics; Vol. v. 1). Beijing: Science Press. (2002).

[35] Martínez, S. An optimization problem with volume constraint in Orlicz spaces. J. Math. Anal. Appl., 2008 vol. 340 p. 1407-1421. 
[36] Martínez, S. and Wolanski, N. A minimum problem with free boundary in Orlicz Spaces. Advances in Mathematics, 218, (2008), no. 6, 1914-1971.

[37] Mikayelyan, H. and Shahgholian, H. Hopfs lemma for a class of singular/degenerate PDEs. Ann. Acad. Sci. Fenn, 40, 475-484 (2015).

[38] Montenegro, M. Strong maximum principles for supersolutions of quasilinear elliptic equations. Nonlinear Analysis 37 (1999) 431-448.

[39] Oliveira, K. and Teixeira, E.V. An optimization problem with free boundary governed by a degenerate quasilinear operator. Diff. Integral Equations 19 (2006), 1061-1080.

[40] Orlicz, W. Ueber eine gewisse Klasse von Räumen vom Typus. Bull. Intern. Acad. Pol. Ser. A , 8/9 (1932) pp. 207-220.

[41] Rao, M. and Ren, Z. Theory of Orlicz spaces, volume 146 of Pure and Applied Mahtematics. Marcel Dekker, Inc., 1991.

[42] Rossi, J.D. Approximation of the Sobolev trace constant. Divulgaciones Matematicas. Vol. 11(2), 109-113, (2003).

[43] Rossi, J. Elliptic problems with nonlinear boundary conditions and the Sobolev trace theorem. In Handbook of differential equations: Stationary partial differential equations (Vol. 2, pp. 311-406). North-Holland.

[44] Sperner Jr., E. Spherical Symmetrization and Eigenvalue Estimates. Math. Z. 176, 75-86 (1981).

[45] Sokolowski, J. and Zolésio, J.-P. Introduction to shape optimization. Springer Series in Computational Mathematics, vol. 16, Springer-Verlag, Berlin, 1992, Shape sensitivity analysis.

[46] Teixeira, E.V. Optimal design problems in rough inhomogeneous media. Existence theory. Amer. J. Math. 132 (2010), 1445-1492.

(J.V da Silva) Departamento de Matemática FCEyn - Universidad de Buenos Aires and IMAS - CONiCET. Ciudad Universitaria, Pabellón I (C1428EGA) Av. Cantilo 2160. Buenos Aires, Argentina.

E-mail address: jdasilva@dm.uba.ar

$U R L:$ https://www.researchgate.net/profile/Joao_Da_Silva12

(A.M. Salort) Departamento de Matemática FCEyN - Universidad de Buenos Aires and IMAS - COniCEt. Ciudad Universitaria, Pabellón I (C1428EGA) Av. Cantilo 2160. Buenos Aires, Argentina.

E-mail address: asalort@dm.uba.ar

URL: http://mate.dm.uba.ar/〜asalort/

(A. Silva) Departamento de Matemática, Universidad Nacional de San Luis and IMASL - CONiCET. Ejército de los Andes 950 (D5700HHW), San Luis, Argentina.

E-mail address: acsilva@unsl.edu.ar

$U R L:$ https://analiasilva.weebly.com

(J.F. Spedaletti) Departamento de Matemática, Universidad Nacional de San Luis and imASL - CONiCET. Ejército de los Andes 950 (D5700HHW), San Luis, Argentina.

E-mail address: jfspedaletti@unsl.edu.ar 